\title{
Protective Role of Aerobic Exercise Against Cisplatin-Induced Nephrotoxicity in Rats
}

\author{
Farzaneh Zeynali, ${ }^{1,2}$ Mehdi Nematbakhsh, ${ }^{1,3,4}{ }^{,}$Hossain Mojtahedi, ${ }^{2}$ Aliasghar Poorshahnazari, ${ }^{3}$ Ar- \\ deshir Talebi, ${ }^{1,5}$ Zahra Pezeshki, Safoora Mazaheri, and Fatemeh Moslemi ${ }^{1}$
}

${ }^{1}$ Water and Electrolytes Research Center, Isfahan University of Medical Sciences, Isfahan, IR Iran

${ }_{3}^{2}$ Department of Physical Education, Faculty of Physical Education, Isfahan University, Isfahan, IR Iran

3 Department of Physiology, Isfahan University of Medical Sciences, Isfahan, IR Iran

4 Department of Physiology, Isfahan University of Medical Sciences, Isfahan, IR

${ }_{5}^{5}$ Department of Clinical Pathology, Isfahan University of Medical Sciences, Isfahan, IR Iran

${ }^{*}$ Corresponding author: Mehdi Nematbakhsh, Water and Electrolytes Research Center, Department of Physiology, Isfahan University of Medical Sciences, Isfahan, IR Iran. Tel: +98-3137922419, Fax: +98-3137922419, E-mail: nematbakhsh@med.mui.ac.ir

Received: July 1, 2014; Accepted: October 22, 2014

\begin{abstract}
Background: Cisplatin (CP) is a chemotherapy drug and nephrotoxicity is considered as its major side effect. Aerobic exercise is well known as an approach to reduce the side effects of many drugs.

Objectives: This study was designed to determine the protective role of aerobic exercise against CP-induced nephrotoxicity. Materials and Methods: Thirty male Wistar rats were randomly divided into four groups. Group I had aerobic exercise on a treadmill one hour per day and five days per week for eight weeks. Then, the exercise protocol was continued for another week, but during this week, the animals also received $\mathrm{CP}(2.5 \mathrm{mg} / \mathrm{kg} /$ day; ip). Group II underwent the same protocol as group I without exercise in the last week during the CP therapy. Groups III and IV were assigned as positive and negative control groups, and were treated with CP and saline without exercise, respectively. Finally, the animals were sacrificed for the biochemical measurement and tissue histopathology investigation.

Results: $\mathrm{CP}$ alone without exercise increased serum levels of blood urea nitrogen (BUN), creatinine(Cr), and malondialdehyde(MDA); and kidney nitrite level, while treadmill exercise in group I significantly ameliorated these parameters $(\mathrm{P}<0.05)$. Kidney and serum levels of MDA and nitrite did not alter significantly. Also, the severity of kidney tissue damage decreased significantly in groups I and II $(\mathrm{P}<0.05)$. Conclusions: Aerobic exercise may reduce CP-induced nephrotoxicity with a favorable effect on renal function by increasing activation of antioxidant system.
\end{abstract}

Keywords: Exercise; Cisplatin; Nephrotoxicity; Rats

\section{Background}

Cancer is a major cause of morbidity and mortality throughout the world (1). Cancer treatment is directly related to the type and stage of the disease, age and medical history of the patient. Chemotherapy is one of the most common treatments $(2,3)$ and cisplatin $(\mathrm{CP})$ as a member of the platinum drugs is used to treat testicular, ovarian, breast, lung, and bladder cancers (4). $\mathrm{CP}$ has numerous side effects such as liver and testis injury (5). The major side effect of CP is nephrotoxicity (6). $\mathrm{CP}$ is the inhibitor of desoxyribonucleic acid synthesis (4) and nephrotoxicity of CP seems to be related to reactive oxygen species. Reactive oxygen species, especially the hydroxyl radical, induce lipid peroxidation and cell membrane damage and reduce glomerular filtration rate (GFR) (7-9). It is reported that CP-induced nephrotoxicity may be ameliorated by scavenging agents such as vitamins E and C, losartan, melatonin, selenium, superoxide dismutase, and erythropoietin (10-16). Researches indicate that exercise activity changes antioxidant content (17-19), and as an example, it reduces antioxidant levels of erythrocyte GSH-PX (17). Also, moderate regular exercise decreased malondialdehyde (MDA) levels (20). Another study showed long-term aerobic exercise increases glutathione erythrocyte peroxidase enzyme (GSH) activity and superoxide dismutase (SOD) activity (18). Physical exercise has been suggested in cancer patients undergoing chemotherapy $(21,22)$ to improve mental and physical activities and rehabilitation, because exercise increases heart-respiratory fitness and muscular strength, and improves body composition. Moreover, exercise reduces fatigue, anxiety, and depression in these patients (23). Accordingly and considering the beneficial effects of exercise, it seems that exercise can affect CP-induced nephrotoxicity. 


\section{Objectives}

The current study was designed to investigate the protective effect of aerobic exercise against CP-induced nephrotoxicity.

\section{Materials and Methods}

\subsection{Experimental Protocol}

Thirty adult male (weighing $211 \pm 7$ gr) Wistar rats (Animal Centre, Isfahan University of Medical Sciences, Isfahan, Iran) were used in this research. The animals were given free access to water and rat chow and housed at a temperature of $23-25^{\circ} \mathrm{C}$ under a 12 hours light/ 12 hours dark schedule. The experimental procedures were approved in advance by the Isfahan University of Medical Sciences Ethics Committee.

The rats were randomly assigned to four groups. The animals in group I (called EX + CP + EX) had aerobic exercise on a treadmill one hour per day and five days per week for eight weeks. Then, the exercise protocol was continued for another week, but during this week, the animals also received CP (2.5 mg/kg/day; ip). Group II (called EX + $\mathrm{CP}$ ) had protocol the same as group I without exercise in the last week during CP therapy. Groups III and IV were assigned as positive (CP) and negative control (called sham) groups, and they were treated with $\mathrm{CP}$ and saline, respectively, without exercise. The animals in the control groups were placed in the apparatus for the same duration of time without running.

\subsection{Treadmill Exercise}

The rats were subjected to treadmill exercise as a moderate regular exercise on a motorized rodent treadmill (Isfahan University of Medical Sciences). To this end, the animals were placed on the apparatus and forced to run at the speed of $16 \mathrm{~m} / \mathrm{min}$ for 15 minutes for 7 days. The animals that could not run were excluded from the study. After one week of adaptation, groups I and II received a progressive exercise. During the first two weeks, the speed was increased to $20 \mathrm{~m} / \mathrm{min}$ for $60 \mathrm{~min} /$ day for 5 days/week, the third and the fourth weeks to $23 \mathrm{~m} / \mathrm{min}$, the fifth and the sixth weeks to $25 \mathrm{~m} / \mathrm{min}$, and the last two weeks to $28 \mathrm{~m} / \mathrm{min}$. After endurance training for eight weeks, group I with reduced aerobic exercise to $23 \mathrm{~m} / \mathrm{min}$ and group II with stopped aerobic exercise were treated with CP for one week. The angle of inclination was $0 \%$ during the study. This method was moderate exercise and it is mentioned that oxygen consumption in this position was about $65 \%$ for the rats $(24,25)$.

\subsection{Measurement and Histopathological Proce- dures}

All animals were weighed during treatment with CP.
At the end of the experiment, the rats were anesthetized with chloral hydrate injection (450 mg/kg; ip) and blood samples were taken from the heart. Then, the animals were sacrificed and right and left kidneys were removed and weighed. The blood samples were centrifuged at $6000 \mathrm{rpm}$. Then, serum was collected and stored at $-20^{\circ} \mathrm{C}$ until measurement. The left kidney was fixed in $10 \%$ neutral formalin solution and embedded in paraffin for histopathological staining. The assessment was conducted by a pathologist who was blinded to study protocol. The changes in kidney tissues were recorded using a grading scale of 0 - 4 which was based on a subjective impression of the changes (tubular dilatation and simplification, tubular cell swelling and necrosis, tubular casts, and intraluminal cell debris with infiltration of inflammatory cells). The kidney tissue damage score (KTDS) was assigned as zero score for normal tissue, score of 1 for $\leq 25 \%$ damage, score of 2 for $>25 \%$ and $\leq 50 \%$ damage, score of 3 for $>50 \%$ and $\leq 75 \%$ damage, and score of 4 for $>75 \%$ tissue damage. The right kidney was homogenized and centrifuged. Then, the supernatant was stored at $-20^{\circ} \mathrm{C}$ for measurement. The levels of serum creatinine $(\mathrm{Cr})$ and blood urea nitrogen (BUN) were determined using quantitative diagnostic kits (Pars Azmoon, Iran). The serum and kidney levels of nitrite (stable NO metabolite) was measured using a colorimetric assay (ELISA) that involves the Griess reaction. The serum and renal levels of MDA were measured manually (26). Bodyweight (BW) of the animals was also measured during the last week of $\mathrm{CP}$ therapy.

\subsection{Statistical Analyses}

Data were expressed as mean \pm SEM. One-way ANOVA (followed by the LSD as post test) was applied to compare the BUN, Cr, NO, and MDA levels; and kidney weight (KW) and bodyweight (BW) between the groups. To compare the KTDS between the groups, Kruskal Wallis and MannWhitney tests were applied. $\mathrm{P}<0.05$ was considered statistically significant.

\section{Results}

\subsection{Effect of CP and Exercise on Serum Levels of $B U N$ and $\mathrm{Cr}$}

The serum levels of BUN and Cr significantly increased in the positive control group (CP group) when compared with the negative control group (sham) $(\mathrm{P}<0.05)$. These parameters were significantly reduced in EX $+\mathrm{CP}+\mathrm{EX}$ group when compared with the positive control group $(\mathrm{P}<0.05)$, but by stopping exercise in group II during CP therapy, no beneficial effect was seen on serum levels of BUN and $\mathrm{Cr}$ (Figure 1). 


\subsection{Effect of CP and Exercise on BW Changes, KW, and KTDS}

$\mathrm{CP}$ alone reduced $\mathrm{BW}$ in the positive control group when compared with the negative control group (sham) $(\mathrm{P}<0.05)$. Exercise had no significant effect on BW. The KW significantly increased in the positive control group when compared with the sham group $(\mathrm{P}<$ 0.05). No significant changes in KW were observed in groups I and II in comparison with the negative and positive control groups. KTDS was evaluated and scored by a pathologist. Higher kidney damage score was detected in the positive control group when compared with the negative control group $(\mathrm{P}<0.05)$. The KTDS in $\mathrm{EX}+\mathrm{CP}+\mathrm{EX}$ and $\mathrm{EX}+\mathrm{CP}$ groups significantly reduced when compared with the positive control group $(\mathrm{P}<$ 0.05 ) (Figure 1). The images of kidney tissues is shown in Figure 2.

\subsection{Effect of $C P$ and Exercise on Nitrite and MDA Levels}

The serum level of nitrite did not alter significantly between the groups. This is while the kidney level of nitrite reduced significantly $(\mathrm{P}<0.05)$ in all $\mathrm{CP}$-treated groups when compared with the sham group. However, this reduction was more in EX + CP + EX group, and accordingly the kidney level of nitrite in group I was significantly reduced by exercise when compared with the positive control group $(\mathrm{P}<0.05)$ (Figure 3$)$.

The serum levels of MDA significantly increased in the positive control group (CP) when compared with the negative control group ( $\mathrm{sham})(\mathrm{P}<0.05)$. Exercise could ameliorate the serum level of MDA in both EX + CP + EX and $\mathrm{EX}+\mathrm{CP}$ groups $(\mathrm{P}<0.05)$. However, no significant change in kidney MDA level was observed between all the experimental groups (Figure 3).

Figure 1. Serum Levels of Blood Urea Nitrogen (BUN) and Creatinine (Cr), Kidney Weight (KW), Bodyweight Change ( $\triangle$ BW), and Kidney Tissue Damage Score (KTDS)
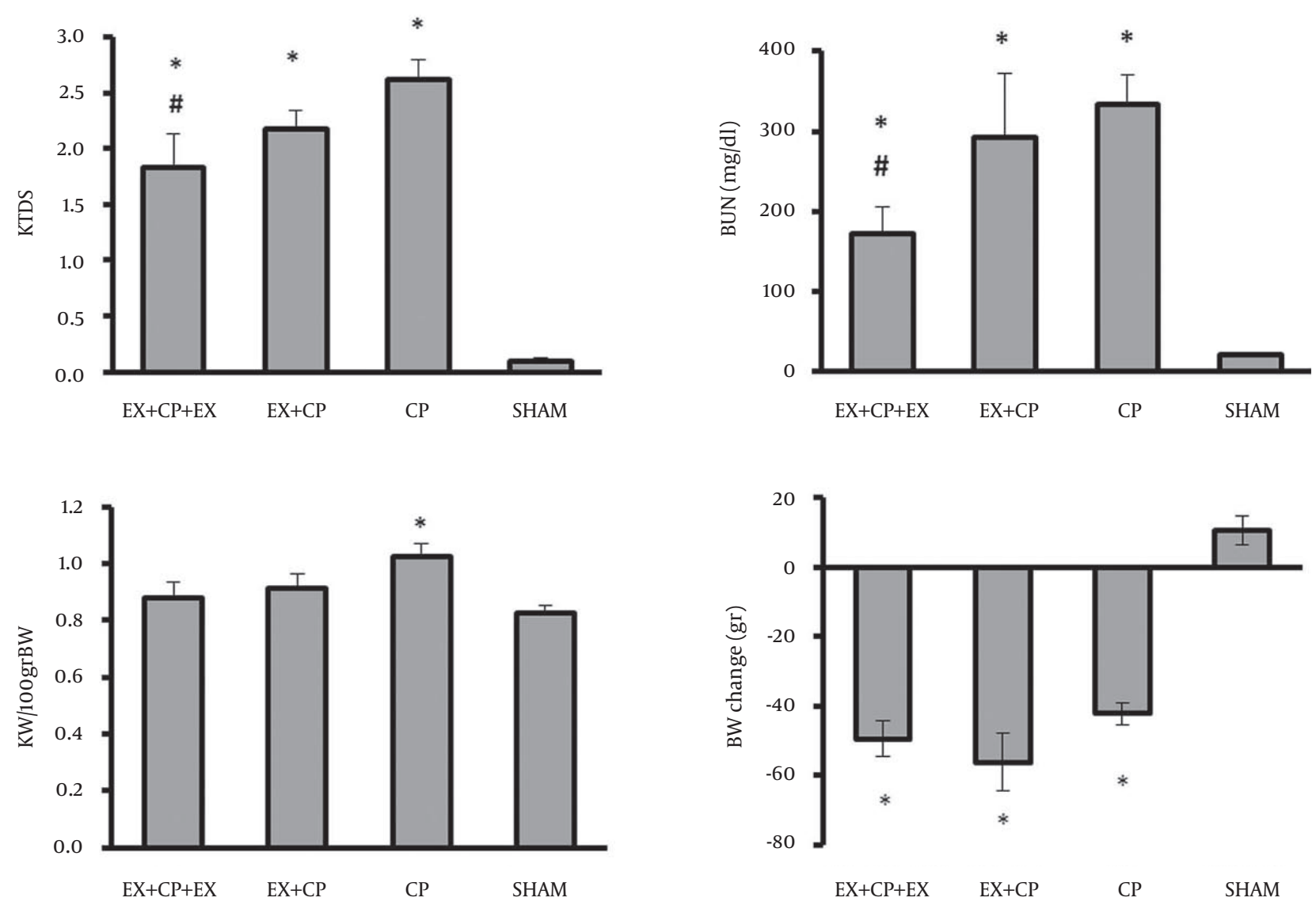

*Indicates significant difference from the negative control group and \# indicates significant difference from the positive control group ( $<<0.05)$. Abbreviations: CP, cisplatin; EX, exercise. 
Figure 2. The Images $(100 \times)$ of Kidney Tissue Stained With Hande to Examine Tissue Damage in the Kidney of The Four Experimental Groups
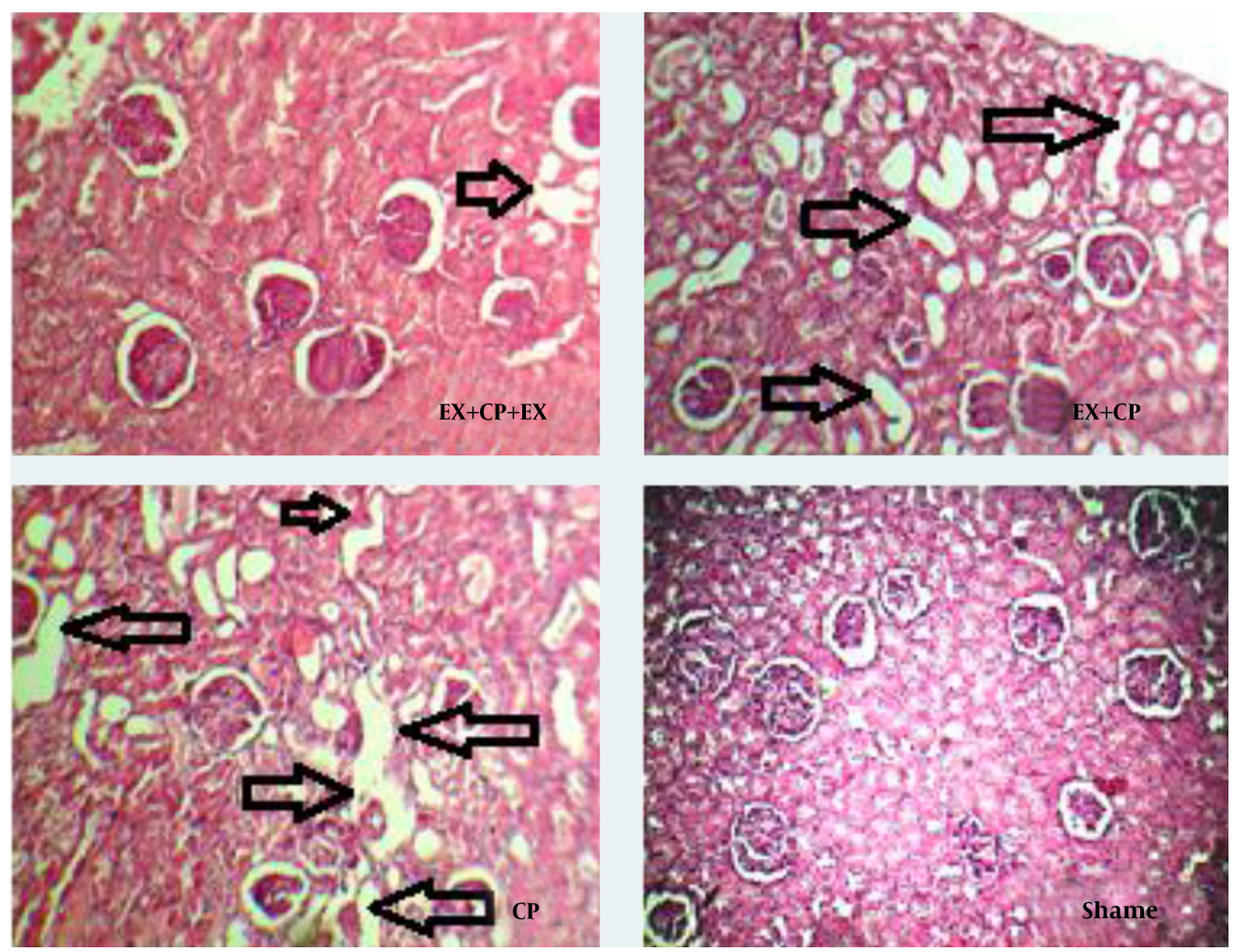

The arrow shows the kidney tissue damage. CP, cisplatin; EX, exercise

Figure 3. Serum Levels of Nitrite and Malondialdehyde (MDA), and Kidney Tissue Levels of Nitrite and MDA
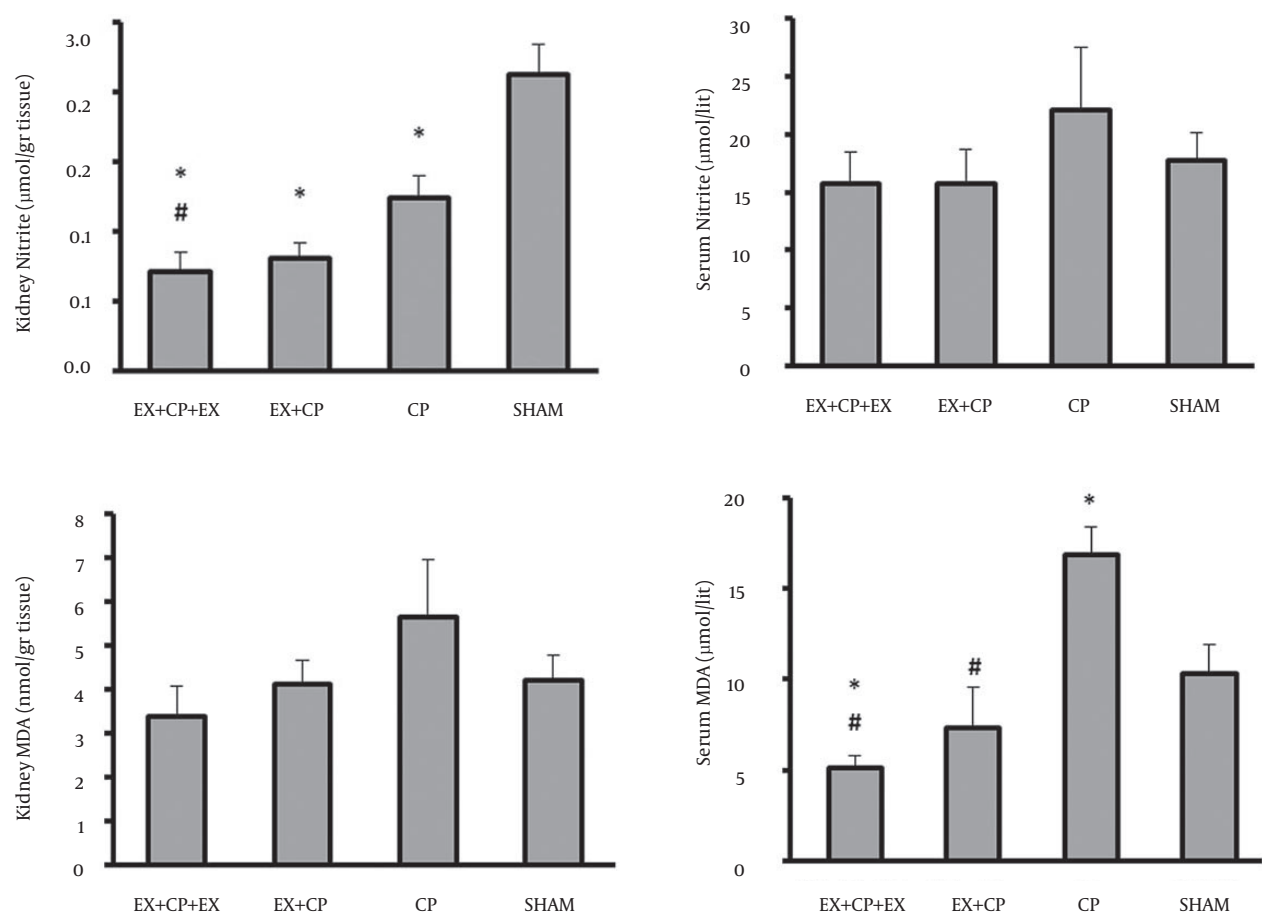

${ }^{*}$ Indicates significant difference from the negative control group and \# indicates significant difference from the positive control group $(\mathrm{P}<0.05)$. Abbreviations: CP, cisplatin; EX, exercise. 


\section{Discussion}

The main objective of this study was to investigate the role of aerobic exercise in reducing CP-induced nephrotoxicity. In our study, CP increased serum levels of Cr, BUN, which was confirmed by pathological data. Our findings were in agreement with previous studies (14, 27-31). Histopathological examination showed that $\mathrm{CP}$ induces massive degenerative changes in 50 - 75\% of glomeruli and renal tubules (32) by mechanisms such as oxidative stress and apoptosis, and our histopathological investigation indicated that moderate regular exercise reduced KTDS. Accordingly, BUN and $\mathrm{Cr}$ were also decreased by exercise. It has been reported before that exercise can improve kidney function by improving metabolic factors and glomerular filtration rate and reduction of albuminuria (33-35). It seems that exercise during $\mathrm{CP}$ therapy could reduce the CP-induced oxidative stress and prevent renal dysfunction. Moreover, exercise potentially could improve endothelial function and CP is known as a drug that causes endothelial injury and impaired endothelial function by decreasing eNOS (36). However, our data did not support this idea when the serum level of nitrite was analyzed. The CP alone therapy not only decreased the serum nitrite level but also increased it insignificantly. Possibly, the increased NO metabolite (nitrite) by CP was not related to eNOS, and it may be produced from other NO formation pathways such as iNOS. Different data for nitrite level in the kidney tissue have been found. We observed that CP decreased the kidney level of nitrite and exercise decreased it more. Therefore, we can conclude that exercise in a CP-treated animal potentially could decrease both serum and kidney levels of nitrite when compared with the non-exercise group, but the importance should be defined in future studies. In our study, CP injection increased the serum MDA level and moderate regular exercise improved this factor. It is reported that appropriate exercise increases antioxidant enzymes (37) and decreases MDA level (20, 38). Furthermore, treatment with antioxidants reduces CP-induced nephrotoxicity $(13,14,27,30,36,39)$. It seems that the positive effect of aerobic exercise on antioxidant defense system can reduce CP-induced nephrotoxicity. In spite of the beneficial effect of moderate regular exercise on kidney function, it had no beneficial effect on body weight. A study showed that physical activity decreased body weight (4042), and it is reported that administration of CP increased kidney weight, which is directly related to kidney injury (43).Our study indicated physical exercise either before CP therapy or simultaneously with $\mathrm{CP}$ therapy may have positive effects on reducing the nephrotoxicity induced by $\mathrm{CP}$. Aerobic exercise may reduce $\mathrm{CP}$-induced nephrotoxicity with a favorable effect on renal function or increasing activation of antioxidant system.

\section{Funding/Support}

This research was supported by Isfahan University of Medical Sciences.

\section{References}

1. Ries F, Klastersky J. Nephrotoxicity induced by cancer chemotherapy with special emphasis on cisplatin toxicity. Am J Kidney Dis. 1986;8(5):368-79.

2. Espandiari P, Zhang J, Rosenzweig BA, Zhou Y, Vaidya VS, Schnackenberg L, et al. Age-Related Differences in Cisplatin-Induced Renal Toxicity in Rats. Faseb J. 2008;22(1):917.

3. Vansteenkiste J, Vandebroek J, Nackaerts K, Dooms C, Galdermans D, Bosquee L, et al. Influence of cisplatin-use, age, performance status and duration of chemotherapy on symptom control in advanced non-small cell lung cancer: detailed symptom analysis of a randomised study comparing cisplatin-vindesine to gemcitabine. Lung Cancer. 2003;40(2):191-9.

4. Hanigan MH, Devarajan P. Cisplatin nephrotoxicity: molecular mechanisms. CancerTher. 2003;1:47-61.

5. Fahmy H. Effect of Low Radiation Dose on Cisplatin Induced Hepato- Testicular Damage in Male Rats. Br J Pharm Res. 2014;4(9):1053-66.

6. Nematbakhsh M, Nasri H. The effects of vitamin E and selenium on cisplatin-induced nephrotoxicity in cancer patients treated with cisplatin-based chemotherapy: A randomized, placebocontrolled study.J Res Med Sci. 2013;18(7):626-7.

7. Satoh M, Kashihara N, Fujimoto S, Horike H, Tokura T, Namikoshi T, et al. A novel free radical scavenger, edarabone, protects against cisplatin-induced acute renal damage in vitro and in vivo. J Pharmacol Exp Ther. 2003;305(3):1183-90.

8. Sugihara K, Nakano S, Koda M, Tanaka K, Fukuishi N, Gemba M. Stimulatory effect of cisplatin on production of lipid peroxidation in renal tissues.Jpn J Pharmacol.1987;43(3):247-52.

9. Winston JA, Safirstein R. Reduced renal blood flow in early cisplatin-induced acute renal failure in the rat. Am J Physiol. 1985;249(4 Pt 2):F490-6.

10. Ali BH, Al Moundhri MS. Agents ameliorating or augmenting the nephrotoxicity of cisplatin and other platinum compounds: a review of some recent research. Food Chem Toxicol. 2006;44(8):1173-83.

11. Nematbakhsh M, Ashrafi F, Safari T, Talebi A, Nasri H, Mortazavi $\mathrm{M}$, et al. Administration of vitamin E and losartan as prophylaxes in cisplatin-induced nephrotoxicity model in rats. J Nephrol. 2012;25(3):410-7.

12. Ban $M$, Hettich D, Huguet N. Nephrotoxicity mechanism of cis-platinum (II) diamine dichloride in mice. Toxicol Lett. 1994;71(2):161-8.

13. Ajith TA, Abhishek G, Roshny D, Sudheesh NP. Co-supplementation of single and multi doses of vitamins $\mathrm{C}$ and $\mathrm{E}$ ameliorates cisplatin-induced acute renal failure in mice. Exp Toxicol Pathol. 2009;61(6):565-71.

14. Ajith TA, Usha S, Nivitha V. Ascorbic acid and alpha-tocopherol protect anticancer drug cisplatin induced nephrotoxicity in mice: a comparative study. Clin Chim Acta. 2007;375(1-2):82-6.

15. Miller RP, Tadagavadi RK, Ramesh G, Reeves WB. Mechanisms of Cisplatin nephrotoxicity. Toxins (Basel). 2010;2(11):2490-518.

16. Hayashi M, Numaguchi M, Watabe H, Enomoto H, Yaoi Y. Cisplatin-induced nephrotoxicity and the protective effect of fosfomycin on it as demonstrated by using a crossover study of urinary metabolite levels. Acta Obstet Gynecol Scand. 1997;76(6):590-5.

17. Duthie GG, Robertson JD, Maughan RJ, Morrice PC. Blood antioxidant status and erythrocyte lipid peroxidation following distance running. Arch Biochem Biophys. 1990;282(1):78-83.

18. Shin YA, Lee JH, Song W, Jun TW. Exercise training improves the antioxidant enzyme activity with no changes of telomere length. Mech Ageing Dev. 2008;129(5):254-60.

19. Berzosa C, Cebrian I, Fuentes-Broto L, Gomez-Trullen E, Piedrafita E, Martinez-Ballarin E, et al. Acute exercise increases plasma total antioxidant status and antioxidant enzyme activities in untrained men.J Biomed Biotechnol. 2011;2011:540458.

20. Naziroglu M, Simsek M, Kutlu M. Moderate exercise with a dietary vitamin $C$ and $\mathrm{E}$ combination protects against streptozotocin-induced oxidative damage to the blood and improves fetal outcomes in pregnant rats. Clin Chem Lab Med. 2004;42(5):511-7.

21. Jacobsen PB, Phillips KM, Jim HS, Small BJ, Faul LA, Meade CD, et al. Effects of self-directed stress management training and 
home-based exercise on quality of life in cancer patients receiving chemotherapy: a randomized controlled trial. Psychooncology. 2013;22(6):1229-35.

22. Knutsen L, Quist M, Midtgaard J, Rorth M, Adamsen L. Maximum physical capacity testing in cancer patients undergoing chemotherapy: qualitative findings from an exercise program. Scand $J$ Med Sci Sports. 2006;16(6):403-11.

23. Doyle C, Kushi LH, Byers T, Courneya KS, Demark-Wahnefried W, Grant B, et al. Nutrition and physical activity during and after cancer treatment: an American Cancer Society guide for informed choices. CA Cancer J Clin. 2006;56(6):323-53.

24. Baranowski M, Zabielski P, Blachnio-Zabielska AU, Harasiuk D, Gorski J. LXR activation prevents exhaustive exercise-induced hypoglycaemia and spares muscle glycogen but does not enhance running endurance in untrained rats. Acta Physiol (Oxf). 2011;201(3):373-9.

25. Powers SK, Criswell D, Lawler J, Martin D, Lieu FK, Ji LL, et al. Rigorous exercise training increases superoxide dismutase activity in ventricular myocardium. Am J Physiol. 1993;265(6 Pt 2):H2094-8.

26. Jilanchi S, Nematbakhsh M, Bahadorani M, Talebi A, Eshraghi-Jazi $\mathrm{F}$, Mansouri A, et al. Vitamin e is a nephroprotectant agent in male but not in female in a model of Cisplatin-induced nephrotoxicity. ISRN Nephrol. 2013;2013:280395.

27. Antunes LM, Darin JD, Bianchi MD. Protective effects of vitamin c against cisplatin-induced nephrotoxicity and lipid peroxidation in adult rats: a dose-dependent study. Pharmacol Res. 2000;41(4):405-11.

28. De Martinis BS, Bianchi MD. Effect of vitamin C supplementation against cisplatin-induced toxicity and oxidative DNA damage in rats. Pharmacol Res. 2001;44(4):317-20.

29. Deegan PM, Nolan C, Ryan MP, Basinger MA, Jones MM, Hande KR. The role of the renin-angiotensin system in cisplatin nephrotoxicity. Ren Fail. 1995;17(6):665-74.

30. Saleh S, Ain-Shoka AA, El-Demerdash E, Khalef MM. Protective effects of the angiotensin II receptor blocker losartan on cisplatin-induced kidney injury. Chemotherapy. 2009;55(6):399-406.

31. Stakisaitis D, Dudeniene G, Jankunas RJ, Grazeliene G, Didziapetriene J, Pundziene B. Cisplatin increases urinary sodium excretion in rats: gender-related differences. Medicina (Kaunas). 2010;46(1):45-50.

32. Aleisa AM, Al-Majed AA, Al-Yahya AA, Al-Rejaie SS, Bakheet SA, AlShabanah OA, et al. Reversal of cisplatin-induced carnitine defi- ciency and energy starvation by propionyl-L-carnitine in rat kidney tissues. Clin Exp Pharmacol Physiol. 2007;34(12):1252-9.

33. Moinuddin I, Leehey DJ. A comparison of aerobic exercise and resistance training in patients with and without chronic kidney disease. Adv Chronic Kidney Dis. 2008;15(1):83-96.

34. Corpeleijn E, Bakker SJ, Stolk RP. Obesity and impaired renal function: potential for lifestyle intervention? Eur J Epidemiol. 2009;24(6):275-80.

35. Boor P, Celec P, Behuliak M, Grancic P, Kebis A, Kukan M, et al Regular moderate exercise reduces advanced glycation and ameliorates early diabetic nephropathy in obese Zucker rats. Metabolism. 2009;58(11):1669-77.

36. Saleh S, El-Demerdash E. Protective effects of L-arginine against cisplatin-induced renal oxidative stress and toxicity: role of nitric oxide. Basic Clin Pharmacol Toxicol. 2005;97(2):91-7.

37. Moien-Afshari F, Ghosh S, Elmi S, Rahman MM, Sallam N, Khazaei $\mathrm{M}$, et al. Exercise restores endothelial function independently of weight loss or hyperglycaemic status in $\mathrm{db} / \mathrm{db}$ mice. Diabetologia. 2008;51(7):1327-37.

38. Alessio HM, Goldfarb AH. Lipid peroxidation and scavenger enzymes during exercise: adaptive response to training. $J$ Appl Physiol (1985). 1988;64(4):1333-6.

39. Weijl NI, Elsendoorn TJ, Lentjes EG, Hopman GD, Wipkink-Bakker A, Zwinderman AH, et al. Supplementation with antioxidant micronutrients and chemotherapy-induced toxicity in cancer patients treated with cisplatin-based chemotherapy: a randomised, double-blind, placebo-controlled study. Eur J Cancer. 2004;40(11):1713-23.

40. Slentz CA, Duscha BD, Johnson JL, Ketchum K, Aiken LB, Samsa GP, et al. Effects of the amount of exercise on body weight, body composition, and measures of central obesity: STRRIDE--a randomized controlled study. Arch Intern Med. 2004;164(1):31-9.

41. Gao Y, Wang C, Pan T, Luo L. Impact of metformin treatment and swimming exercise on visfatin levels in high-fat-induced obesity rats. Arq Bras Endocrinol Metabol. 2014;58(1):42-7.

42. Kenney WL, Wilmore J, Costill D. Physiology of Sport and Exercise With Web Study Guide-5th Edition.United States of America: Human Kinetics; 1999. p. 710.

43. Nematbakhsh M, Ashrafi F, Nasri H, Talebi A, Pezeshki Z, Eshraghi $\mathrm{F}$, et al. A model for prediction of cisplatin induced nephrotoxicity by kidney weight in experimental rats. J Res Med Sci. 2013;18(5):370-3. 